LIVER

\title{
Which patients with primary biliary cirrhosis or primary sclerosing cholangitis should undergo endoscopic screening for oesophageal varices detection?
}

\author{
B Bressler, R Pinto, D El-Ashry, E J Heathcote
}

Gut 2005;54:407-410. doi: 10.1136/gut.2004.040832

\begin{abstract}
See end of article for authors' affiliations

Correspondence to: Dr E J Heathcote, Toronto Western Hospital, University Health Network, 399 Bathurst Street, 6th Fl Fell Pavilion $\mathrm{Rm} 170$

Toronto, Ontario M5T 2S8, Canada; jenny.heathcote@ utoronto.ca
\end{abstract}

Revised version received 3 August 2004

Accepted for publication 14 August 2004
Background: Recent guidelines from an AASLD Single Topic Symposium suggest that patients with cirrhosis, including those with primary biliary cirrhosis (PBC) or primary sclerosing cholangitis (PSC), should be screened for oesophageal varices when the platelet count is $<140000 / \mathrm{mm}^{3}$.

Aim: To determine the validity of these guidelines in clinical practice in patients with PBC or PSC. Methods: Retrospective review of individuals undergoing screening upper endoscopy for oesophageal varices at a single centre. Oesophageal varices were reported as being present or absent.

Results: A total of 235 patients with chronic liver disease, including 86 patients with PBC $(n=79)$ or PSC $(n=7), 104$ patients with chronic viral hepatitis, and 45 with non-alcoholic cirrhosis of differing aetiologies, underwent a single screening endoscopy between 1996 and 2001 . Oesophageal varices were detected in $26(30 \%)$ of the PBC/PSC group, $38(37 \%)$ of the viral hepatitis group, and $21(47 \%)$ of the "other" group. Applying multiple logistic regression analysis to the data in the group with PBC/PSC, platelets $<200000 / \mathrm{mm}^{3}$ (odds ratio (OR) 5.85 (95\% confidence interval (Cl) 1.79-19.23)), albumin

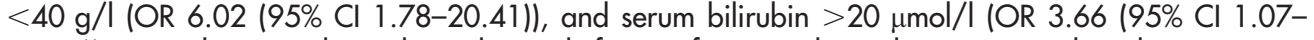
12.47)) were shown to be independent risk factors for oesophageal varices. Prothrombin time was unhelpful. The values at these cut offs were not useful in predicting oesophageal varices in the other groups.

Conclusion: We conclude that current guidelines recommended by the AASLD Single Topic symposium are invalid in our cohort of patients with PBC and PSC. Patients with a platelet count $<200000 / \mathrm{mm}^{3}$, an albumin level $<40 \mathrm{~g} / \mathrm{l}$, and a bilirubin level $>20 \mu \mathrm{mol} / \mathrm{l}$ should be screened for oesophageal varices.
$\mathrm{S}$ everal studies indicate the efficacy of primary preventive therapy with non-selective $\beta$-blockers to reduce the risk of haemorrhage from oesophageal varices. ${ }^{1}$ Variceal haemorrhage is a serious complication of portal hypertension which causes a high mortality rate. The presence of oesophageal varices occurs most commonly as a consequence of cirrhosis. The mortality rate after a variceal bleed has been estimated to be $21 \%$ within one month of the event. ${ }^{2}$

In 1996, a published report of the AASLD Single Topic Symposium on portal hypertension recommended screening for oesophageal varices in patients with Child's class A cirrhosis when there are signs of portal hypertension, specifically: a platelet count $\left(140000 / \mathrm{mm}^{3}\right)$ and/or enlarged portal vein diameter $(>13 \mathrm{~mm})$, or in patients with Child's class B or $\mathrm{C}$ at the time of diagnosis. ${ }^{1}$ Schepis et al subsequently performed a cross sectional study to develop a predictive model for the presence of oesophageal varices using these recommendations. ${ }^{3}$ Their results validated these AASLD recommendations, and Schepis et al showed that independent variables that predicted the presence of oesophageal varices were: platelet count $<100 \times 10^{3}$, portal vein diameter $>13 \mathrm{~mm}$, and prothrombin activity $<70 \%{ }^{3}$ The aetiology of liver disease in this cross sectional study was predominately chronic viral hepatitis $(77.7 \%)$; in only $1.4 \%$ was the classification autoimmune liver disease.

Individuals with primary biliary cirrhosis (PBC) or primary sclerosing cholangitis (PSC) may present de novo with variceal haemorrhage early in the course of their disease and even in the absence of established cirrhosis. ${ }^{45}$ This is thought to be due to portal tract inflammation causing localised vascular damage leading to venous thromboses within the liver resulting in presinusoidal portal hypertension. Gores and colleagues ${ }^{6}$ followed 265 patients with
PBC for an average of 5.6 years, who at entry into the study had no evidence of oesophageal varices. They noted that $31 \%$ developed varices over this time period and $48 \%$ had one or more episodes of variceal bleeding. Although the risk of bleeding if varices were present was the same as for other causes of liver disease, survival after the bleeding episode was better in those with $\mathrm{PBC}$, one and three year survival rates being $65 \%$ and $46 \%$, compared with a one year survival of only $34 \%$ in alcohol related cirrhosis. ${ }^{7}$ The explanation given was that patients with $\mathrm{PBC}$ were more likely to have preserved hepatic function at the time that they developed portal hypertension. More recently it has been shown that the point prevalence for portal hypertension in patients with PBC was 25\%, ${ }^{8}$ and that the one year overall survival of patients with $\mathrm{PBC}$ with evidence of oesophageal varices was only $63 \%$, regardless of bleeding history. ${ }^{9}$

The appropriate time to begin screening for oesophageal varices in patients with $\mathrm{PBC}$ is unclear and clinical parameters which suggest the presence of oesophageal varices may differ from those identified in patients with chronic viral induced disease reported by Schepis et al. As a confident clinical diagnosis of $\mathrm{PBC}$ can be established without a liver biopsy (antimitochondrial antibody positive, with elevated alkaline phosphatase levels), the stage of liver disease may be unknown and therefore it is particularly relevant to establish guidelines using non-invasive parameters.

The aim of this study was to determine if specific noninvasive measures (platelet count, serum albumin, prothrombin time, or serum bilirubin) were predictors of the presence

Abbreviations: PT, prothrombin time; PBC, primary biliary cirrhosis; PSC, primary sclerosing cholangitis; ROC, receiver operating characteristic; $\mathrm{OR}$, odds ratio 
of oesophageal varices in patients with either PBC or PSC, and whether the predictors were the same as for individuals with cirrhosis due to chronic viral hepatitis or other nonalcohol related causes.

\section{METHODS}

Retrospective review was performed of all consecutive patients undergoing gastrointestinal endoscopy for screening for oesophageal varices from 1996 to 2001 at the Toronto Western Hospital, a tertiary referral centre for liver disease. Subjects were eligible if they either had a diagnosis of cirrhosis based on liver biopsy or ultrasound or a diagnosis of $\mathrm{PBC}$ or PSC. All patients given a new diagnosis of cirrhosis, PBC, or PSC routinely undergo screening endoscopy for oesophageal varices. Patients with advanced cirrhosis (ChildPugh class C), previous or current history of variceal haemorrhage, hepatocellular carcinoma, history of alcohol related liver cirrhosis, current alcohol abuse, current treatment with $\beta$ blockers, or infection with human immunodeficiency virus were excluded. Those with cirrhosis due to alcohol were excluded because of the direct effect alcohol could have on the patient's platelet count. Laboratory parameters examined were from blood tested within three months of the procedure. One experienced endoscopist performed all gastrointestinal endoscopies and was unaware of the abdominal examination findings and laboratory results at the time of endoscopy. A criterion for screening for oesophageal varices in patients with cirrhosis was based on their diagnosis of cirrhosis, regardless of laboratory results. On initial evaluation of all patients diagnosed with $\mathrm{PBC}$ or PSC, endoscopy for variceal screening was performed regardless of histological states. The presence or absence of oesophageal varices was recorded together with the size of the varices according to the grade I-IV classification. ${ }^{10}$ The presence or absence of portal gastropathy was also recorded in the PBC/PSC group.

Data were retrospectively collected based on predetermined variables. Endoscopic findings together with clinical and laboratory variables were entered into a computerised database. Patients were divided into three groups based on the aetiology of their liver disease: PBC/PSC, compensated cirrhosis due to viral hepatitis, or compensated cirrhosis from other non-alcohol related liver disease ("other"). We selected platelet count, serum albumin, bilirubin, and prothrombin time (PT) as possible predictors of the presence of oesophageal varices on endoscopy. The Mayo risk score was also calculated for patients with PBC to determine if it too could predict the presence of oesophageal varices on endoscopy. The research ethics board at the University Health Network, Toronto, Canada, approved the study protocol.

\section{Statistical analysis}

Statistical significance of each variable was assessed as a continuous variable. The sensitivity and specificity of each significant variable was estimated by a receiver operating characteristic (ROC) curve. The curve shows the ability of the variable to distinguish between patients with oesophageal varices and those without. The larger area under the ROC curve the better the discriminating ability of the rule. For each significant variable a cut off point was selected based on the variable's ROC curve such that the overall error rate was low taking into account its sensitivity and specificity.

Stepwise logistic regression was used to determine the relationship between the presence of oesophageal varices and the aforementioned variables in patients in all three groups and between the presence of portal gastropathy and these variables in the PBC/PSC group. Variables in the multiple logistic regression analysis were also used to create a probability prediction rule in the PBC/PSC group. One of the measures of the predictive performance is a measure of concordance, the $c$ index, that for logistic regression is identical to area under the ROC curve. Internal validity was assessed by the bootstrap method. ${ }^{11}$ A bootstrap sample is obtained by sampling with replacement from the original sample. Therefore, a bootstrap sample may contain a patient $0,1,2, \ldots, n$ times where $n$ is the size of the original sample. Based on the bootstrap samples one can make inference about the population from which the original sample was drawn. Bootstrap validation was compared with other validation methods and recommended as it produces stable estimates and low bias. ${ }^{12} 13$

The model for oesophageal varices in PBC/PSC was validated as follows.

(1) Draw a bootstrap sample and perform a logistic regression, selecting the covariates according to stepwise selection criterion. Estimate the logistic regression coefficients, and calculate the $c$ index.

(2) Calculate p, the predicted probabilities, for the original data, using the bootstrap coefficients above. Fit the logistic model in the original data: $y=\alpha+\beta \times \log (p /$ $(1-p)) . \beta$ is called the calibration slope.

(3) Calculate the $c$ index in the original data but using the bootstrap coefficients from step 1 .

Steps 1-3 were repeated 100 times. We averaged over the calibration slopes calculated in step 2 to obtain the shrinkage factor. Regression coefficients are multiplied by the shrinkage factor for the final predictive model to improve predictions in future patients. The intercept in the final model is such that predictions agree on average with the observed frequency of the outcome. The difference in the average $c$ index in step 1 and average $c$ index in step 3 is the optimism index.

\section{RESULTS}

A total of 236 patients who met all of the inclusion/exclusion criteria underwent at least one screening oesophagogastroduodenoscopy between 1996 and 2001. There were 86 individuals with a diagnosis of either PBC or PSC (77 of these patients had PBC), 104 had cirrhosis due to chronic viral hepatitis, and 45 had another form of non-alcohol related compensated cirrhosis (table 1). Patient age, sex,

Table 1 Clinical and biochemical parameters of patients

\begin{tabular}{lccc}
\hline Variable & PBC/PSC $(\mathbf{n}=\mathbf{8 6})$ & Viral $(\mathbf{n}=104)$ & Other $(\mathbf{n}=\mathbf{4 5})$ \\
\hline Age $(\mathrm{y})$ & $56(11)$ & $52(12)$ & $57(16)$ \\
Female $(\%)$ & 89 & 37 & 69 \\
Platelets $\left(\times 10^{3} / \mathrm{mm}^{3}\right)$ & $217.6(82.1)$ & $131.2(64.5)$ & $148.5(82.8)$ \\
Albumin $(\mathrm{g} / \mathrm{l})$ & $42.5(7.1)$ & $41.65(6.0)$ & $39.91(6.6)$ \\
Bilirubin $(\mu \mathrm{mol} / \mathrm{l})$ & $23.2(25.4)$ & $20.8(11.3)$ & $25.7(21.5)$ \\
Prothrombin time (s) & $12.7(2.6)$ & $13.4(2.0)$ & $13.2(1.9)$ \\
\hline \multicolumn{4}{l}{} \\
Values are mean (SD). & & \\
PBC, primary biliary cirrhosis; & PSC, primary sclerosing cholangitis.
\end{tabular}


Table 2 Platelet count cut offs for each different patient group

\begin{tabular}{ll}
\hline Group & Platelet count $\left(/ \mathrm{mm}^{3}\right)$ \\
\hline PBC/PSC $(n=86)$ & $<200000(5.85,1.79-19.23)^{*}$ \\
Viral $(n=104)$ & $<110000(4.42,1.89-10.31)^{*}$ \\
Other $(n=45)$ & $<105000(5.49,1.39-21.74)^{*}$
\end{tabular}

*Odds ratio (95\% confidence interval).

PBC, primary biliary cirrhosis; PSC, primary sclerosing cholangitis.

platelet count, serum albumin, serum bilirubin, and prothrombin time are described in table 1. The aetiology of cirrhosis in patients in the "other" liver disease control group were: autoimmune hepatitis, cryptogenic, haemochromatosis, methotrexate induced, non-alcoholic steatohepatitis, and Wilson's disease.

Oesophageal varices were detected in $26(30 \%)$ patients with PBC/PSC, in $38(37 \%)$ of those with cirrhosis due to chronic viral hepatitis, and in 21 (47\%) of the "other" group. Within the group with PBC/PSC, 11 of 26 patients had oesophageal varices $\geqslant$ grade 2 , in the chronic hepatitis group 16 of 38 patients had oesophageal varices $\geqslant$ grade 2 , and in the "other" group of cirrhosis 10 of 20 had oesophageal varices $\geqslant$ grade 2 .

In the group with PBC/PSC, univariate logistic analysis for the presence of varices indicated that platelets, albumin, bilirubin, Mayo risk score, and patient age were all significantly associated with the presence of oesophageal varices. Because the Mayo risk score includes albumin, bilirubin, and age, correlations between the Mayo risk score and these variables have a magnitude between 0.45 and 0.66 and therefore it was not included in the multiple regression analysis. Cut off values were derived based on their specificity, sensitivity, and lowest total error rate according to their ROC curves: platelet count curve AUC 0.79 (SEM 0.06); serum albumin curve AUC 0.78 (SEM 0.06); and bilirubin curve AUC 0.75 (SEM 0.06).

Multiple logistic regression indicated that platelet count $<200000 / \mathrm{mm}^{3}$ (odds ratio (OR) $5.85 \quad$ (95\% confidence interval (CI) 1.79-19.23)) (table 2), albumin $<40 \mathrm{~g} / \mathrm{l}$ (OR $6.02(95 \%$ CI $1.78-20.41)$ ), and serum bilirubin $>20 \mu \mathrm{mol} / \mathrm{l}$ (OR 3.66 (95\% CI 1.07-12.47)) were the only independent statistically significant parameters which were predictive of the presence of oesophageal varices. The overall $\chi_{3}^{2}=33.91$ $(p<0.0001)$ and the Hosmer and Lemeshow goodness of fit test had a value of $0.3156(p=0.9888)$, indicating the model's adequacy for these data. In our case, the average calibration slope, used as a shrinkage factor for the regression coefficients, was 0.88 . The probability of oesophageal varices, as determined based on the original data, was $88 \%$ in those individuals with a platelet count $<200000 / \mathrm{mm}^{3}$, serum albumin $<40 \mathrm{~g} / \mathrm{l}$, and bilirubin $>20 \mu \mathrm{mol} / \mathrm{l}$ (table 3 ). The adjusted probabilities after the coefficients were shrank (table 3) did not differ greatly from the predicted probabilities in the original data. In our case, the average bootstrap $c$ index was 0.87 and the average $c$ index in the original data was 0.84 , therefore the optimism was 0.03. A model predicting the presence of oesophageal varices $\geqslant$ grade 2 was not possible due to the low numbers of PBC/PSC patients in this group. Also, because of the low number of patients with PSC in our cohort, we were unable to determine if these results would be valid in only the subgroup of patients with PSC.

In the group with cirrhosis due to chronic viral hepatitis, univariate logistic analysis for the presence of varices indicated that platelets, albumin, bilirubin, and prothrombin time were significant factors. Multiple logistic regression indicated that the only examined variable which reliably predicted the presence of detectable oesophageal varices was a platelet count of $<110000 / \mathrm{mm}^{3}$ (OR 4.42 (95\% CI $1.89-$ 10.31), ROC curve AUC 0.72 (SEM 0.06)) (table 2).

In the "other" group, the only examined variable to predict the presence of detectable oesophageal varices was also a low platelet count of $<105000 / \mathrm{mm}^{3}$ (OR 5.49 (95\% CI 1.39 21.74), ROC curve AUC 0.69 (SEM 0.08)) (table 2).

Stepwise logistic regression for the presence of portal gastropathy in the PBC/PSC group indicated that platelet count $<100000 / \mathrm{mm}^{3}$ was the only variable found to be statistically significant (OR11.24 (95\% CI 2.07-62.5), ROC curve AUC 0.7 (SEM 0.07)). Therefore, if the platelet count was $<100000 / \mathrm{mm}^{3}$ in a patient with $\mathrm{PBC} / \mathrm{PSC}$, the probability of having portal gastropathy was $75 \%$, but if the platelet count was $>100000 / \mathrm{mm}^{3}$, the probability of having portal gastropathy was only $21.1 \%$.

\section{DISCUSSION}

Laboratory parameters that were investigated to predict the presence of oesophageal varices in this study are routine blood tests done in most patients with chronic liver disease. Multiple logistic regression indicated that in patients with PBC or PSC, platelet count $<200000 / \mathrm{mm}^{3}$, serum albumin $<40 \mathrm{~g} / \mathrm{l}$, or bilirubin $>20 \mu \mathrm{mol} / \mathrm{l}$ were independent parameters which could reliably predict the presence of oesophageal varices. The ability of these variables to predict whether a patient with PBC/PSC has oesophageal varices had a discriminatory power similar to that indicated in the paper by Schepis and colleagues, ${ }^{3}$ in that each of the variable's AUC for their respective ROC curve was approximately 0.70 . The two major differences from the study of Schepis and colleagues $^{3}$ were the inability of the PT values to help in predicting the presence of oesophageal varices and the higher platelet count found to predict oesophageal varices in our PBC/PSC group but not in patients with cirrhosis due to chronic viral hepatitis. The reason why the PT value was unhelpful could be because, as previously reported, hepatic function in patients with PBC/PSC and oesophageal varices is often preserved.

Table 3 Predicted probability of oesophageal varices in patients with primary biliary cirrhosis/primary sclerosing cholangitis depending on biochemical parameters

\begin{tabular}{lllll}
\hline $\begin{array}{l}\text { Platelet } \\
\text { count }\end{array}$ & Albumin & Bilirubin & Probability & $\begin{array}{l}\text { Adjusted } \\
\text { probability }\end{array}$ \\
\hline Low & Low & Low & $67.70 \%$ & $63.65 \%$ \\
Low & Low & High & $88.45 \%$ & $84.56 \%$ \\
Low & High & Low & $25.82 \%$ & $26.50 \%$ \\
High & Low & Low & $26.39 \%$ & $27.01 \%$ \\
Low & High & High & $55.99 \%$ & $53.01 \%$ \\
High & Low & High & 56.72 & $53.66 \%$ \\
High & High & Low & $5.6 \%$ & $7.08 \%$ \\
High & High & High & $17.87 \%$ & $19.26 \%$ \\
\hline
\end{tabular}


There are several possible explanations why the cut off value for platelets was higher in PBC/PSC patients with oesophageal varices than in patients with other liver diseases complicated by cirrhosis. The presence of thrombocytopenia in patients with portal hypertension occurs by three possible mechanisms. Splenic sequestration and antibody mediated destruction of platelets are known to occur in patients with cirrhosis. ${ }^{14}{ }^{15}$ Moreover, thrombopoietin, a thrombocytopoietic growth factor, is produced in the liver and diminished production plays a significant role in reducing platelets in patients with cirrhosis. ${ }^{16}{ }^{17}$ It may be that the production of thrombopoietin is preserved in PBC/PSC patients with good liver function and this in turn may lead to a higher platelet count cut off when predicting the presence of oesophageal varices.

The natural history of PBC can be reliably predicted using non-invasive investigations. Liver biopsy in PBC may only be performed, if at all, at the time of initial presentation more for prognostic than for diagnostic purposes. Serial biopsies are not routinely performed for staging purposes. The Mayo risk score has been validated as a reliable guide to disease progression in PBC.

Recommendations previously stated by the AASLD, ${ }^{1}$ and supported by a prospective study by Schepis and colleagues, ${ }^{3}$ have provided clinicians with reasonable guidelines based on clinical, biochemical, and radiographic measurements as to when one should begin endoscopic screening for the presence of oesophageal varices with cirrhosis. Our results also concur with these guidelines in both the cirrhosis due to chronic viral hepatitis group and the "other" cirrhosis group but not in patients with PBC or PSC. Our results suggest that screening for oesophageal varices is needed at a much higher cut off for platelet count in PBC/PSC. Zein et al at the Mayo Clinic have recently confirmed this finding in patients with PSC. ${ }^{18}$ They suggested that a platelet count of $<150000 \mathrm{~mm}^{3}$ would be a satisfactory marker for selecting patients with PSC who are likely to benefit from endoscopy screening for oesophageal varices. Portal gastropathy in patients with PBC/ PSC occurred at a similar platelet count value as has been reported in patients with other diseases causing cirrhosis and portal hypertension. Clinical and biochemical predictors of portal gastropathy have not been previously described in patients with PBC/PSC. Our study showed that a platelet count $<100000 / \mathrm{mm}^{3}$ in this patient populations will predict the presence of this condition.

The issue of when to begin and the appropriate interval for screening endoscopy is also more complicated in patients with PBC/PSC. The AASLD recommends that patients with Child's class A who have no evidence of oesophageal varices on initial screening should undergo a repeat endoscopy at two years. Our results suggest one could delay repeat endoscopy in patients with PBC/PSC if they have the following biochemical values: platelet count $>200$ 000/ $\mathrm{mm}^{3}$, serum albumin $>40 \mathrm{~g} / \mathrm{l}$, or bilirubin $<20 \mu \mathrm{mol} / \mathrm{l}$.
Based on our results, the probability of varices being present in a patient if they have all three of these values is only $5.6 \%$.

The non-invasive indicators which indicate that screening endoscopy be performed in all patients with cirrhosis were invalid in our cohort of PBC/PSC patients. Values for the noninvasive indicators from this study should ultimately be validated in a prospective study before being used to determine which patients with PBC or PSC should undergo oesophageal variceal screening endoscopy.

\section{Authors' affiliations}

B Bressler, R Pinto, D El-Ashry, E J Heathcote, University Health Network, Toronto Western Hospital, University of Toronto, Toronto, Canada

Conflict of interest: None declared.

\section{REFERENCES}

1 Grace ND, Groszman RJ, Garcia-Tsao G, et al. Portal hypertension and variceal bleeding: an AASLD single topic symposium. Hepatology 1998:28:868-80.

2 El-Serag HB, Everhart JE. Improved survival after variceal hemorrhage over an 11 -year period in the Department of Veterans Affairs. Am J Gastroenterol 2000;95:3566-73.

3 Schepis F, Calogero C, Niceforo D, et al. Which patients with cirrhosis should undergo endoscopic screening for esophageal varices detection? Hepatology 2001;33:333-8.

4 Colina F, Pinedo F, Solis JA, et al. Nodular regenerative hyperplasia of the liver in early histological stages of primary biliary cirrhosis. Gastroenterology, 1992;102, 1319-24.

5 Kew MC, Varma RR, Dos Santos HA, et al. Portal hypertension in primary biliary cirrhosis. Gut 1971;12:830-4.

6 Gores GJ, Wiesner RH, Dickson ER, et al. Prospective evaluation of esophageal varices in primary biliary cirrhosis: development, natural history and influence on survival. Gastroenterology 1989;96:1552-9.

7 Graham DY, Smith JL. The course of patients after variceal hemorrhage. Gastroenterology 1981;80:800-9.

8 Jones DEJ, Walter R, Prince $\mathrm{Ml}$, et al. The prevalence of portal hypertension in primary biliary cirrhosis (PBC). Gut 2002;50:A113.

9 Jones DEJ, Walter R, Prince MI, et al. The impact of portal hypertension in primary biliary cirrhosis (PBC). Gut 2002;50:A118.

10 Beppu K, Inokuchi K, Koyanagi N, et al. Prediction of variceal hemorrhage by esophageal endoscopy. Gastrointest Endosc 1981;27:213-18.

11 Efron B, Tibshirani R. An introduction to bootstrap. New York: Chapman and Hall, 1993.

12 Steyerberg E, Harrell FE Jr, Borsboom GJJM, et al. Internal validation of predictive models: Efficiency of some procedures for logistic regression analysis. J Clin Epidemiol 2001;54:774-81.

13 Steyerberg E, Eijkemans MJC, Harrell FE Jr, et al. Prognostic modeling with logistic regression analysis: in search of a sensible strategy in small data sets. Med Decis Making 2001;21:45-56.

14 Toghill P, Green S, Ferguson R. Platelet dynamics in liver disease with special reference to the role of the spleen. J Clin Pathol 1977;30:367-71.

15 Tumen HJ. Hypersplenism and portal hypertension. Ann NY Acad Sci 1970;170:332-7

16 Peck-Radosavljevic M, Zacherl J, Meng YG, et al. Is inadequate thrombopoietin production a major cause of thrombocytopenia in cirrhosis of the liver? J Hepatol 1997;27:127-31.

17 Martin TG, Somberg KA, Meng YG, et al. Thrombopoietin levels in patients with cirrhosis before and after orthotopic liver transplantation. Ann Intern Med 1997; 127:285-8

18 Zein CO, Lindor KD, Angulo P. Prevalence and predictors of esophageal varices in patients with primary sclerosing cholangitis. Hepatology 2004;39:203-9. 